\title{
Crisis económica, post pandemia y el problema laboral
}

\section{Economic crisis, post pandemic and the labor problem}

\author{
Ángel Estigarribia ${ }^{a}$
}

\begin{abstract}
Resumen
Es clave comprender las causas fundamentales de la crisis económica actual y sus implicaciones en el mundo del trabajo. El Coronavirus es un detonante, pero no la razón de la crisis. Después de cada crack económico sobreviene una serie de medidas de reorganización productiva que afecta profundamente las condiciones laborales. Las instituciones internacionales y los gobiernos no ocultan sus planes de reformas. Desde la revolución conservadora de Reagan no ha parado el proceso de flexibilización, que con cada crisis se ha profundizado.
\end{abstract}

Palabras clave: crisis económica, pandemia, reforma laboral.

\begin{abstract}
Understanding the root causes of the current economic crisis and its implications for the world of work is key. The Coronavirus is a trigger but not the reason for the crisis. After each economic crash, a series of productive reorganization measures ensues that profoundly affects working conditions. International institutions and governments do not hide their reform plans. Since Reagan's conservative revolution, the process of flexibility has not stopped, which has deepened with each crisis.
\end{abstract}

Keywords: economic crisis, pandemic, labor reform.

\section{Introducción}

La pandemia del COVID-19 fue la chispa que inició el incendio de una crisis económica que hace rato se temía y cuyos elementos generadores se perfilaban. Salió a la superficie un conjunto de problemas políticos, económicos y sociales del que la pandemia es un síntoma. La rápida y letal expansión responde a causas que no son médicas. La Organización Mundial de la Salud (OMS) advirtió después de la "gripe aviar" en el 2003 que era inminente una epidemia de alcance mundial y que, eventualmente por factores diversos, su proliferación iba a ser muy rápida (ONU, 2003). a Universidad Nacional de Asunción, Facultad de Ciencias Sociales, Paraguay.

Correspondencia a: aestigarribia@yahoo.com

Recibido:

15 de mayo de 2020

Aceptado:

29 de junio de 2020

Artículo publicado en acceso abierto bajo la Licencia Creative Commons.

\section{(c) (i)}

Cita:

Estigarribia, A. (2020). Crisis económica, post pandemia y el problema laboral. Kera Yvoty: reflexiones sobre la cuestión social, 5 (número especial), 134-141. 
La OMS recomendó invertir en los sistemas de salud, pero por la crisis económica del 2008, la mayoría de los gobiernos hicieron lo contrario, discontinuaron los programas sanitarios e incluso, ejecutaron planes de austeridad que afectaron profundamente los sistemas de salud y de seguridad social.

$\mathrm{Al}$ inicio de la pandemia y la crisis económica del 2020, la prioridad de las principales potencias ha sido auxiliar el sistema financiero. Las medidas económicas realizadas por la superpotencia mundial, EE.UU. que aprobó el más grande paquete de ayuda de su historia, en su mayor parte se destina a las entidades bancarias, empresas, aerolíneas antes que al sistema de salud (Coronavirus. EE.UU. aprueba un paquete de ayudas económicas de US\$2 billones, el más grande de su historia. 2020, 27 de marzo).

Las cifras de contagios y muertos son inéditas en este sigloy existe un gran margen de desconocimiento de la envergadura real dada las tomas restringidas de muestras y el subregistro de decesos (Wallace, 2020). El sub registro se debe a varios factores (Camilla Costa \& Cecilia Tombesi, 12 mayo 2020) no solo los problemas estrictamente estadísticos, sino también sociales. Los trabajadores pobres, las minorías raciales discriminadas, los indocumentados cuando enferman no van a los servicios, en algunos países porque no tienen dinero, en otros por temor a la deportación, y en casi todos por los dos motivos. También, cuando mueren, no se registran las causas.

En América Latina, la situación de salud de los trabajadores es aún peor, por las condiciones más precarias de los servicios, la desinversión en salud de los últimos años, la pobreza creciente y el decrecimiento económico generalizado (Kurmanaev et al, 2020) y sobre todo, por la abrupta caída del empleo que conlleva una inmediata pauperización de grandes masas.

\section{Las causas profundas}

La economía mundial no ha terminado de recuperarse de la crisis del 2008 (Tabanera, 2020) cuando se sumerge de nuevo en un pozo más profundo. A todas luces la recaída se estaba incubando. "Durante la crisis mundial de 2008, la deuda pública de Latinoamérica alcanzaba el 40\% del PIB. Para el año 2019, antes de la emergencia sanitaria, la deuda pública interna y externa latinoamericana era, en promedio, del 62\% del PIB" (Morales, Oliva \& Oglieti, 2020).

La pandemia del COVID-19, es un disparador y una causa secundaria por la disminución de la actividad fabril y comercial, pero no la causa principal de la actual recesión mundial. La crisis económica es una característica genética del sistema capitalista,

Según el Fondo Monetario Internacional, desde 1970 hasta finales de 2008 se han producido en el mundo 447 crisis financieras nacionales (124 crisis bancarias, 208 de devaluación de la moneda, 63 de la deuda externa del país, 42 dobles y 10 triples) (Dávila \& González, 2009).

La profundización de los problemas económicos viene desde el crack del 2008. El Coronavirus ha encendido la mecha, pero no es la dinamita de la explosión. Ninguna crisis de esta envergadura es producto de una razón coyuntural o de un accidente circunstancial.

Martín Wolf, del Financial Times y un economista marxista, Michael Roberts, desde distintos presupuestos y por distintos caminos llegan a la misma conclusión: el problema de fondo es el exceso de capital que tiene hambre de ganancias.

Estamos en una "situación de catástrofe de la que posiblemente no nos recuperemos realmente por décadas" (Wolf, 2020). Podríamos terminar en "la depresión económica más grande de la historia" (Wolf, 2020) Esta crisis económica actual proviene de un "exceso de ahorro de los ricos" (Wolf, 2020), es decir, una inmensa cantidad de capital que dejó de invertirse productivamente.

La economía desde el 2008 se 
sostenía merced a una expansión de la demanda sustentada en el endeudamiento de las corporaciones, de los estados y de los hogares en todo el mundo, que hizo aumentar y mantener el consumo pero que ha llegado un punto en el que colapsa, porque no aumenta la inversión productiva sino la especulativa (Berggruen \& Gardels, 2013), existe mucho dinero que no tiene relación con la producción real.

En la misma línea afirma Roberts (10 de Mayo de 2020). El endeudamiento que ya venía muy sobredimensionado será agudizado por la pandemia pero esta no la provoca, solo es sino la desencadenante de la crisis de acumulación de la deuda corporativa, estatal y de los hogares.

Marx señala que el capitalismo tiene un problema estructural producto de su propio dinamismo al que llamó ley de la tendencia decreciente de la tasa de ganancia (Marx, 1995, p. 213) La única manera de crear riqueza y acumularla es a través de la extracción de plusvalía del trabajo vivo, de la labor del trabajador. Con la tecnificación, el aumento de la productividad crece exponencialmente, así un trabajador que con una máquina $(\mathrm{x})$ produce 100 cosas por día, con una nueva tecnología puede producir 1000 y luego incluso 10.00o. Pero sucede que, en cada cosa producida, a medida que se multiplica la producción, disminuye el aporte del trabajo vivo y aumenta el aporte del trabajo muerto (máquinas y herramientas, insumos, tecnología), y, por lo tanto, de cada producto se saca menos plusvalía. Esto explica el abaratamiento de los productos cuando se hace masivo, pero también la tendencia decreciente de la ganancia.

Por otro lado, la enorme masa de dinero que circula de manera especulativa por el mundo, sumado al volumen estratosférico de ganancias que sobreviene de la flexibilización laboral, las privatizaciones, la incorporación de masas laborales en condiciones precarias y baratas hacen que el volumen de capital que busca ganancias se multiplique, pero al no aumentar la tasa de ganancia, no tiene donde invertir, lo que provoca el "exceso de ahorro" del que habla Wolf.

"La profunda caída de 1980-1982 diezmó los sectores manufactureros en el norte global" (Roberts, 10 de Mayo de 2020). Los capitales de las potencias del norte entonces se convirtieron en deuda externa de los países pobres del sur con lo que rápidamente volvieron las ganancias y una abrupta acumulación del capital. "Se invirtieron los beneficios y el dinero prestado en bonos y acciones lo que redujo las tasas de interés y aumentó las ganancias de capital y los precios de las acciones. Las empresas lanzaron un programa interminable de recompra de sus propias acciones para aumentar los precios de las acciones y pidieron prestado para hacerlo" (Roberts, 10 de Mayo de 2020).

El acceso al crédito barato endeudó al mundo entero, gobiernos, empresas, familias. La tarjeta de crédito se hizo masiva, llegando hasta a los sectores más vulnerables.

Lo que puso fin a este auge crediticio especulativo fue la disminución de la rentabilidad del capital desde fines de la década de 1990, que condujo al pinchazo de la burbuja de "alta tecnología" de 2001 y, finalmente, al colapso financiero y a la Gran Recesión de 2008. El "exceso de ahorro" es en realidad el otro lado de una "escasez de inversión” (Roberts, 2020).

De la baja rentabilidad de los activos productivos se pasó a una burbuja especulativa alimentada por la deuda en activos ficticios. "Las crisis no son el resultado de un déficit de "demanda endeudada": son causadas por un déficit de rentabilidad" (Roberts, 2020).

El capital ganancioso busca inversiones para ganar más, si baja la tasa de ganancia en la producción deja de invertir en ella y va a la especulación financiera hasta que estalla la burbuja. El retiro del capital de la esfera productiva lleva al desempleo masivo y a la crisis.

\section{El sacrificio de los trabajadores}

La crisis económica de la década 
del 8o tuvo su correlato en las políticas de flexibilización laboral y privatizaciones de empresas públicas impulsada por los más importantes organismos multinacionales como FMI y el Banco Mundial a iniciativa de EE.UU. y el Reino Unido y, finalmente, a partir de los 9o, en la incorporación a la esfera capitalista de la mano de obra calificada y barata proveniente de la exURSS y de China, que constituyeron el salvataje del sistema.

A medida que aumenta la productividad, decrece la tasa de plusvalía, para tratar de recuperar, el capital tiene lo que Marx denominó "fuerzas contrarrestantes de la ley de la tendencia decreciente de la tasa de ganancia" (Marx, 1995, p. 232), de las cuales se destacan tres: el "aumento de la tasa de explotación del trabajo" (Marx, 1995, p. 232); "reducción del salario por debajo de su valor" (Marx, 1995 p. 235) y la "superpoblación relativa" (Marx, 1995, p. 236) que deriva en la enorme masa de desocupados que presiona a la baja el nivel salarial.

Las tres causas citadas apuntan a lo mismo: mayor extracción de plusvalía del trabajo vivo. Los grandes cambios en la ingeniería de los procesos laborales tuvieron en vista ese mismo objetivo, tanto el taylorismo como el fordismo y el toyotismo, que correspondieron a momentos muy distintos, el fordismo para satisfacer a un mercando en expansión y la necesidad de la producción masiva, el toyotismo para enfrentar la sobreproducción y la necesidad de responder a mercados más segmentarizados, apuntaron también a debilitar la organización de los trabajadores y trabar la solidaridad para permitir profundizar la extracción de plusvalía (Coriat, 1993).

El toyotismo ha sido la respuesta orgánica de la producción a la crisis de los 70, con la incorporación del sistema justo a tiempo, cero stock, el trabajo en equipo, la planificación participativa de la producción, la polivalencia funcional (Coriat, 1993).

La reingeniería de procesos vino a responder a la crisis de los 80 (Hammer y
Champy, 2005), el estudio cronométrico de los procesos, la eliminación de los tiempos muertos, la plurifunción de los trabajadores.

La polivalencia funcional, la bolsa de horarios, los grupos de calidad total, la planificación participativa, los equipos de trabajo, el pago por colectivos laborales han sido medidas para cambiar la relación de los trabajadores con la empresa y de los trabajadores entre sí.

El capital no solo apela a la incorporación de tecnologías para aumentar la explotación, tendencia que se acelera en tiempos de crisis, también desarrolla dispositivos organizacionales, procesos y protocolos de control. Las reformas en la organización del trabajo llevan a la intensificación de la productividad y con ello a la disminución del costo laboral.

La tendencia más probable en la post-pandemia es la profundización de los sistemas de flexibilización y reingeniería de procesos que venía desde antes del 2008.

- Flexibilización de la jornada de trabajo, del manejo de los horarios y el tiempo libre de los trabajadores. Jornada laboral determinada por las necesidades y ritmos de la producción (incluidos los fines de semana y feriados como días laborales normales). Bolsa de horarios, se utiliza para no pagar horas extras en las jornadas más largas, sino que se compensan con menos horas en otros días.

- Pagoporobjetivosdeproductividad, desaparece el pago por el tiempo de labor, día, semana, quincena o mes.

- Contratos de trabajo sin prestaciones sociales, jubilación, seguro médico, bonificación familiar, ayuda alimentaria, ni vacaciones.

- Contratos por tiempo determinado que no acumulan antigüedad.

- Tercerización, trabajadores desligados de la empresa pero que trabajan en la empresa: servicios de limpieza, reparaciones, 
comida, seguridad, recursos humanos, sistema de pagos, pero también montaje, empaquetado, distribución.

- Terminación de contrato sin indemnización.

La máxima expresión de la flexibilización hoy es Uber. Uberización es el nombre de un fenómeno popularizado por Maurice Lévy “'Uberización' es esa idea que al despertar por la mañana uno descubre que su trabajo ha desaparecido" (Thompson, 2014). La nueva incorporación a la estrategia de control del trabajo es lo que ha venido a llamarse el modelo Uber de explotación laboral.

Por representar un símbolo de un nuevo modelo económico, la firma Uber se prestó para crear esta expresión. Esta expresión se usa para definir los nuevos modelos de negocios en los cuales particulares pueden efectuar transacciones económicas vía plataformas accesibles desde aplicaciones que se encuentran en sus celulares inteligentes o en sus computadores. Además de Uber, los últimos años han experimentado una explosión de oferta de plataformas que van desde el alojamiento con AirBnB hasta plataformas de intermediación financiera, otras dedicadas a los servicios domésticos, a los servicios jurídicos, etc. (Bardey, 2015, parr. 2).

Las plataformas montadas en Internet combinan dos aspectos de los negocios que parecían irreconciliables: la masificación del mercado y la necesidad de personalizar la atención de los clientes. El fenómeno de la sobreproducción había empujado a las empresas a buscar o construir nichos de mercado orientados a compradores de núcleos específicos con necesidades específicas, pero al mismo tiempo quedaba restringida la producción masiva.

Con el sistema Uber se sintetizan ambos extremos. Un cliente puede elegir el modelo y color del automóvil que quiere utilizar, sin los carteles de taxi y sin necesidad de pagar en efectivo, tiene los datos del conductor y sabe exactamente cuánto leva a costary el itinerario a seguir. $\mathrm{Al}$ mismo tiempo, el servicio tiene el respaldo de una gran empresa internacional, con servicios estandarizados en varias partes del mundo. El trabajador no tiene una relación laboral con la empresa, es un "autónomo" que compra el servicio de Uber. Un robot maneja el negocio.

Uber es sólo el principio. Las tecnologías informáticas permiten agrupar en una misma plataforma millones de individuos y al mismo tiempo desplegar un proceso de personalización a gran escala. Miles de empleos podrán ser automatizados. "La Universidad de Oxford pronosticó que el $47 \%$ de los empleos corren el riesgo de ser reemplazados por robots y computadoras con inteligencia artificial en Estados Unidos durante los próximos 15 a 20 años" (Oppenheimer, 2018, p. 11).

Millones de empleos que eran más o menos independientes están siendo incorporados a sistemas parecidos a Uber, como UpWorky Freelancer con lo que pasan a ser dependientes: abogados, arquitectos, diseñadores, desarrolladores, vendedores inmobiliarios, de seguros, técnicos de diversos servicios, médicos, odontólogos, en una rápida asalarización a destajo, sin beneficios sociales tradicionales.

Siempre después de cada crisis, el capital -a través de los gobiernos y organismos internacionales- apunta a sacar mayor plusvalía de los trabajadores como condición para la recuperación.

Por tanto, un mayor grado de flexibilidad laboral permitiría —en teoría - la creación de mayor empleo y la reducción del sector informal, de ahí que dichos organismos han promovido la aplicación de diversos mecanismos de flexibilidad laboral para disminuir el elevado desempleo prevaleciente desde la década de los ochenta del siglo pasado. (González \& Cisneros, 2010, p. 38) 
Los mecanismos son variados pero los discursos que los justifican tienen siempre la misma línea: sacrificio por el país.

Después de la segunda guerra mundial, la burguesía en Francia pedía sacrificios a los trabajadores para poner en pie la economía, hasta la izquierda se vio involucrada en el movimiento, la consigna de Maurice Thorez "Primero producir" era para poner freno a las exigencias sindicales (Judt, T. 2005). Esta consigna se ha repetido de diversas formas innumerables veces para pedir a los trabajadores posponer sus reivindicaciones hasta que la economía se recupere.

En las crisis, el capital no alienta al único productor de riqueza, la clase trabajadora, no da incentivos, no promueve el bienestar ni el mejoramiento de las condiciones de trabajo, ni mucho menos impulsa el empleo masivo de mano de obra, todo lo contrario.

Para algunos organismos internacionales, como el Banco Mundial y la Organización para la Cooperación y el Desarrollo Económico (OCDE), la flexibilidad consiste en eliminar o desregular el mercado laboral con el objetivo de acabar con las rigideces causantes de un alto índice de desempleo, así como de un sector informal creciente. En este sentido, el Banco Mundial sostiene que la principal causa del desempleo en América Latina reside, fundamentalmente, en la inflexibilidad de los mercados laborales, lo que se traduce en que el sector formal de la economía no sea dinámico en términos de generación de empleos. (González \& Cisneros, 2010, p. 38)

El Banco Mundial recomienda, además de la flexibilización laboral, la desmonopolización y la privatización de las empresas estatales, es decir, trasladar los servicios y la producción de bienes que realiza el Estado a la órbita mercantil para beneficios privados.
Durante el período de recuperación, los países deberán calibrar la disminución del apoyo público y dedicar especial atención a los desafíos de desarrollo más amplios. El análisis abarca la importancia de propiciar una asignación ordenada del capital nuevo a sectores que sean productivos en las nuevas estructuras que surjan en el período pos pandémico. Con ese fin, los países deberán incorporar reformas que permitan que el capital y la mano de obra se adapten con relativa rapidez, mediante la aceleración de la resolución de controversias, la disminución de los obstáculos regulatorios y la reforma de los costosos subsidios, monopolios y empresas estatales protegidas que han lentificado el desarrollo (Banco Mundial, 2 de junio de 2020).

\section{Menos solidaridad}

La mitad de los trabajadores del mundo, unos 1.600 millones, podrían perder su trabajo (OIT, 2020) y situarse en la miseria, pero la pérdida del empleo no se debe solamente a la pandemia. Las grandes empresas tecnológicas -salvo Amazon- despedían trabajadores incluso mucho antes de la pandemia, al mismo tiempo han aumentado considerablemente sus ingresos en esta crisis, sobre todo Amazon, Google, Netflix, Zoom, Youtube (Coronavirus: ¿quiénes están ganando dinero con la epidemia? 2020, 4 de marzo).

La catástrofe no nos hace más solidarios. El hambre ya era un problema muy grave antes de la pandemia. El Programa Mundial de Alimentos advertía que, para finales de año "veremos a más de la cuarta parte de mil millones de personas sufriendo hambre aguda en países con bajos y medianos ingresos" (Anthem, 2020). Antes del COVID-19 ya estaban en esta situación 135 millones de individuos. Ahora, centenares de millones de trabajadores ocasionales se han convertido abruptamente en pobres extremos.

Con la crisis y el crecimiento abrupto de la pobreza, la lógica mercantil ha prevalecido sobre todo. Algunos ejemplos 
son bárbaros: las empresas productoras de alimentos en EE.UU., cuando no podían vender sus productos como lácteos o papas, los tiraban a la basura (Fox, L. \& Navarro, A. 2020) porque donarlos supone costos (cadena de frío, transporte, distribución, etc.). La FAO calcula que un tercio de todos los alimentos producidos se tira, sin contar los desperdicios minoristas y de los hogares (Pérdida de alimento, 2020).

\section{Destrucción creativa}

La economía capitalista suele usar varios caminos para enfrentar la crisis, pero los más importantes se reducen a una sola: la destrucción de las fuerzas productivas, la aniquilación de buena parte de las riquezas conseguidas, para reanudar luego la reconstrucción, es lo que Schumpeter (2010) ha llamado destrucción creativa. La guerra, las quiebras, las ofertas hostiles de compra, las fusiones forzadas, la obsolescencia programada (Espeleta, 2017) la destrucción de los recursos naturales aun a sabiendas de los efectos irreversibles son parte de la misma lógica.

En la organización laboral, la destrucción creativa del capitalismo ha sido la normalidad de los últimos 40 años: desregulación de las normativas, precarización, sobreexplotación en especial de las mujeres y de los inmigrantes-, incorporación de niños como fuerza de trabajo, retroceso a condiciones semiesclavistas de vida en amplios rincones del planeta.

Las consecuencias de la crisis económica del 2020 y de la pandemia serán peores que las guerras mundiales que nos antecedieron y las soluciones no pueden ser sino globales, podemos seguir el curso destructivo o emprender cambios cualitativos en las relaciones de producción.

\section{Referencias}

Anthem, P. (2020, 16 de abril ). Risk of hunger pandemic as COVID-19 set to almost double acute hunger by end of 2020. World Food Programme Insight. https://insight.wfp.org/covid-19- will-almost-double-people-in-acutehunger-by-end-of-2020-59dfoc4a8072

Banco Mundial (2020). Los países pueden adoptar medidas ahora para la reconstrucción posterior a la COVID-19. Comunicado de prensa 2 de junio de 2020. https://www.bancomundial.org/ es/news/press-release/2020/o6/o2/ countries-can-take-steps-now-tospeed-recovery-from-covid-19

Bardey, D. (4 de agosto de 2015). ¿Qué significa la "Uberización" de la economía? ¿Cuáles son sus consecuencias para Colombia? Foco Económico. http://focoeconomico. org/2015/o8/o4/que-significala-uberizacion-de-la-economiacuales-son-sus-consecuencias-paracolombia-por-david-bardet/

Berggruen, N., \& Gardels, N. (2013). Gobernanza inteligente para el siglo XXI. Taurus.

Cisneros, M., \& González, L. (2010). La flexibilidad laboral como estrategia de competitividad y sus efectos sobre la economía, la empresa y el mercado de trabajo. Contaduría y Administración, (231).

Coriat, B. (1993). Pensar al revés. Trabajo y organización en la empresa japonesa. Siglo XXI.

Coronavirus. EE.UU. aprueba un paquete de ayudas económicas de US\$2 billones, el más grande de su historia (27 marzo 2020). BBC. https:// www.bbc.com/mundo/noticiasinternacional-52045106

Coronavirus: ¿quiénes están ganando dinero con la epidemia? (4 de marzo de 2020). BBC. https://www.bbc.com/ mundo/noticias-51710459

Costa, C., \& Tombesi, C. (12 mayo de 2020) Coronavirus: cómo se comparan las muertes por covid-19 con las mayores causas de mortalidad en el mundo y en América Latina. BBC News. https:// www.bbc.com/mundo/noticiasinternacional-52591995)

Dávila, C., \& González, B. (2009). Crisis económica y salud. Gaceta Sanitaria, 
23(4), 261-265. https://gacetasanitaria. org/es-crisis-economica-saludarticulo-So213911109001885)

Espeleta, A. (2017). La obsolescencia programada en el bien de consumo y la dinámica del mercado [Trabajo fin de Máster]. Universidad Complutense de Madrid. https://eprints.ucm.es/45668/

FAO. (2020). Pérdida de alimento. http:// www.fao.org/food-loss-and-foodwaste/es/

Hammer, M., Champy, J. (2005). Reingeniería. Ed. Norma.

Judt, T. (2005). Postwar: A History of Europe since 1945. Penguin Press.

Kurmanaev, A., Andreoni, M., Casado, L, Taj, M. (12 de mayo de 2020). Latin America's Outbreaks Now Rival Europes. But its option are Worse. New York Times. https://www.nytimes. com/2020/05/12/world/americas/ latin-america-virus-death.html)

Morales, T., Oliva, N., \& Oglietti, G. (2020, 31 de mayo). COVID-19 y más FMI en América Latina. Sin Permiso.Info. https://www.sinpermiso.info/textos/ covid-19-y-mas-fmi-en-america-latina $31 / 05 / 2020$

OIT. (2020, 29 de abril). Comunicado de prensa. https://www.ilo.org/global/ about-the-ilo/newsroom/news / WCMS_743056/lang--es/index.htm

ONU. (2003). La gripe aviar y la amenaza de pandemia. https://www.un.org/es/ influenza/about.shtml

Roberts, M. (10 de Mayo de 2020). The debt dilema [Entrada en blog]. Michael Roberts Blog. https://thenextrecession. wordpress.com

Roberts, M. (6 de junio de 2020). Returning to normal? [Entrada en blog]. Michael Roberts Blog. https://thenextrecession. wordpress.com/

Schumpeter, J. (2010). Capitalism, Socialism $\mathcal{E}$ Democracy. Tayson \& Francis Group.

Tabanera, N. (7 de abril de 2020) La economía mundial podría tardar siete años en recuperarse. Economía $y$ finanzas. https://www.finanzas. com/economia-politica/la-economiamundial-podria-tardar-siete-anosen-recuperarse-de-la-crisis-delcoronavirus_20051460_102.html

Thompson, A. (14 de diciembre de 2014) Maurice Lévy tries to pick up Publicis after failed deal with Omnicom. Financial Times. https://www.ft.com/ content/377f7054-81ef-11e4-b9doo0144feabdco

Wallace, A. (17 de abril de 2020). Cómo se cuentan los muertos por coronavirus (y por qué las cifras oficiales no son comparables ni precisas). BBC News Mundo. https://www.bbc.com/ mundo/noticias-52313544

Wolf, M. (2020). After the Pandemic [video] Financial Times. https://www. ft.com/video/dafc3578-5c76-412a8ba3-016495031bd1

\section{Sobre el Autor}

Ángel Estigarribia

Licenciado en Filosofía y Magíster en Filosofía Política Contemporánea, Universidad Nacional de Asunción (UNA). Docente de la Facultad de Filosofía y de la Facultad de Ciencias Sociales (UNA). 